\title{
Personalized Pain Goals and Responses in Advanced Cancer Patients
}

\begin{abstract}
Sebastiano Mercadante, MD, * Claudio Adile, MD, ${ }^{*}$ Federica Aielli, MD, ${ }^{\dagger}$ Lanzetta Gaetano, MD, ${ }^{\ddagger, \S}$ Kyriaki Mistakidou, MD, " Marco Maltoni, MD," Luiz Guilherme Soares, MD,"|| Stefano DeSantis, MD,** Patrizia Ferrera, MD,* Marta Rosati, MD, $\|$ Romina Rossi, MD, and Alessandra Casuccio, BS ${ }^{\dagger \dagger}$

*Pain Relief \& Supportive Care, La Maddalena Cancer Center, Palermo, Italy; ${ }^{\dagger}$ Department of Biotechnological and Applied Clinical Sciences, University of L'Aquila, L'Aquila, Italy; ${ }^{\ddagger}$ Medical Oncology Unit, IRCCS Neuromed, Pozzilli, Italy; ${ }^{\S}$ Medical Oncology Unit, Italian Neuro-Traumatology Institute, Grottaferrata, Italy; "Pain Relief and Palliative Care Unit, Department of Radiology, Areteion Hospital, National \& Kapodistrian University of Athens, School of Medicine, Athens, Greece; "Palliative Care Unit, Istituto Scientifico Romagnolo per lo Studio e la Cura dei Tumori (IRST) IRCCS, Meldola, FC, Italy; IIPost-Acute Care Services and Palliative Care Program, Hospital Placi, Niterói, Rio de Janeiro, Brazil; **Palliative Care and Oncologic Pain Service, S. Camillo-Forlanini Hospital, Rome, Italy; ${ }^{\dagger \dagger}$ Department of Sciences for Health Promotion and Mother Child Care, Università di Palermo, Palermo, Italy
\end{abstract}

Correspondence to: Sebastiano Mercadante, MD, La Maddalena Cancer Center, Via San Lorenzo 312, 90145 Palermo, Italy, Tel: 39-0916806521; Fax: 39-091-6806110; E-mail: terapiadeldolore@lamaddalenanet.it; 03sebelle@gmail.com.

Conflict of interest: No conflict of interest to declare.

\begin{abstract}
Objective. To assess the personalized pain intensity goal (PPIG), the achievement of a personalized pain goal response (PPGR), and patients' global impression (PGI) in advanced cancer patients after a comprehensive pain and symptom management. Design. Prospective, longitudinal Setting. Acute pain relief and palliative/supportive care. Subjects. 689 advanced cancer patients. Methods. Measurement of Edmonton Symptom Assessment Score (ESAS) and personalized pain intensity goal (PPIG) at admission (T0). After a week (T7) personalized pain goal response (PPGR) and patients' global impression (PGI) were evaluated. Results. The mean PPIG was 1.33 (SD 1.59). A mean decrease in pain intensity of - 2.09 was required on PPIG to perceive a minimal clinically important difference (MCID). A better improvement corresponded to a mean change of -3.41 points, while a much better improvement corresponded to a mean of -4.59 points. Patients perceived a MCID (little worse) with a mean increase in pain intensity of 0.25 , and a worse with a mean increase of 2.33 points. Higher pain intensity at T0 and lower pain intensity at T7 were independently related to PGI. 207 (30.0\%) patients achieved PPGR. PPGR was associated with higher PPIG at T0 and T7, and inversely associated to pain intensity at T0 and T7, and Karnofsky level. Patients with high pain intensity at T0 achieved a favorable PGI, even when PPIG was not achieved by PPGR. Conclusion. PPIG, PPGR and PGI seem to be relevant for evaluating the effects of a comprehensive management of pain, assisting decision-making process according to patients' expectations. Some factors may be implicated in determining the individual target and the clinical response.
\end{abstract}

Key Words: Cancer Pain; Clinical Response; Pain Intensity

\section{Introduction}

Patients with cancer often experience pain [1]. The prevalence of pain in this population has been estimated to be $>75 \%$ for those with advanced disease [2]. Cancer pain is a complex multidimensional phenomenon. Inadequate pain assessment is one the most relevant barriers to providing appropriate pain management. Thus, patientreported pain and symptom evaluation has been considered the gold standard to assess clinical response. The
Edmonton Symptom Assessment System (ESAS) is one of the most common tools used to evaluate both physical and psychological symptoms. This simple instrument, which is easy to use and repeatable, is based on a unidimensional numeric rating scale ranging from 0 (no symptom) to 10 (worse possible) for each symptom taken into consideration [3]. However, this instrument may have some limitations because patients may individually 
interpret the scale, variably expressing intensity. On the other hand, the clinical response after initiating a particular treatment is difficult to assess, as the minimal clinically important difference (MCID) is often not established. The MCID is considered the smallest amount of change required to impact a patient's feeling of improvement or deterioration after treatment. The MCID has been the subject of recent research. Some tools have been reported as methods to assess MCID, including the distribution method [4-6] and the use of anchors, such as the change of intensity categories of well-being [7], the optimal balance between sensitivity and specificity, and the magnitude of change in outcome reported by the patient. The need to evaluate the individual variations in assessing scales or numbers remains of paramount importance. Thus, the use of the Patient Global Impression (PGI) scale has been suggested. This is a validated global rating-of-change scale to assess patients' subjective response, as it is based on the individual feeling of improvement or deterioration after receiving a drug [8]. Furthermore, a personalized symptom goal has been recently introduced to tailor pain (Patients' Pain Intensity Goal [PPIG]) and symptom management, providing a simple and individualized "target" score on the ESAS $[9,10]$. Therapeutic attempts should try to reach such a threshold for an intrapatient determination of an expected response to any treatment. The concept of Personalized Pain Goal Response (PPGR), which is both practical and meaningful, represents the achievement of the expected PPIG, individually determined. Factors associated with PPIG and PGI have never been examined. Studies have assessed these points, even for pain [7,9-12]. However, data were retrospectively examined or performed in an outpatient setting, with variable intervals for the follow-up. An optimal characterization of PPIG and a study of factors associated with PPGR and PGI, as perceived by patients, would help clinicians to maximally personalize pain management and to evaluate meaningful changes. This is even more important in a palliative care unit, the setting where pain and symptom management can be more rapid and effective because daily assessment, expertise, and timely therapeutic changes may provide better control of pain and symptoms in a short period.

This study was performed to characterize the PPIG, PPGR, and PGI after comprehensive pain management in advanced cancer patients. The secondary aim was to assess the factors that can influence these outcomes.

\section{Methods}

This is a subanalysis of a large international study of advanced cancer patients performed at admission to five palliative care units in Italy, Brazil, and Greece [13]. The ethical committees at all participating centers approved the study. All participants provided written informed consent. Participating centers were tertiary care palliative care units within a comprehensive cancer department.

\section{Participants}

A comprehensive pain and symptom assessment was performed by a specialist palliative care physician. Inclusion criteria were age $\geq 18$ years and a diagnosis of advanced cancer. Exclusion criteria were no pain, a short life expectancy (less than two weeks), cognitive failure (a score of $\geq 13$ on the Memorial Delirium Assessment Scale [MDAS]) [14].

\section{Data Collection}

Patients' characteristics, including age, gender, education level, and cancer diagnosis, were recorded, as well as initial Karnofsky performance status.

Symptom intensities included in the ESAS (pain, shortness of breath, fatigue, nausea, depression, anxiety, drowsiness, insomnia, appetite, feelings of well-being) were measured at admission (T0). The same measurements were performed seven days after starting a comprehensive palliative care treatment (T7). The comprehensive palliative care intervention was based on specialized assessment and treatment of symptoms. No strict protocols were given, and treatments were based on local policy. Researchers participating in the study were experienced in providing palliative care. One week was assumed to be an acceptable time to experience the effects of a clinical intervention.

The ESAS is a self-reported tool assessing the intensity of most common psychological and physical symptoms; it uses a numeric rating scale ranging from 0 (no symptom) to 10 (worst intensity) points to examine symptom intensity over the past 24 hours. It is a valid and reliable tool for assessing the overall symptom burden and is sensitive to changes produced by treatment [3]. A screening tool for history of alcohol dependence (CAGE: cut down, annoy, guilt, eye-opener) was also used, as a positive CAGE score (2) has been variably shown to have prognostic value in opioid management [15].

At T0, patients were asked about their PPIG. The question was: "At what level would you feel comfortable with pain?" using the numeric rating scale used for ESAS [12]. One week (T7) after starting comprehensive pain and symptom management tailored to patients' needs and local policy, ESAS and PPIG were measured to evaluate the changes. Patients achieved the PPGR if their intensity measured at T7 was equal to or less than their expected PPIG. At the same interval (T7), PGI (improvement or deterioration) was measured on a scale from +3 to -3 (much better, better, a bit better, the same, a little worse, worse, and much worse, respectively). PGI has been used assess a clinically significant changes in pain intensity $[12,13]$. The MCID was calculated by PGI at T7 (a bit better or a little worse, respectively).

\section{Statistical Analysis}

Quantitative and qualitative data, including descriptive statistics, were analyzed for all items. Continuous data 
Table 1. Characteristics of patients

\begin{tabular}{|c|c|c|}
\hline \multicolumn{2}{|l|}{ Age, mean (SD) [range], y } & $64.3(12.5)$ [18-97] \\
\hline \multicolumn{2}{|l|}{ Gender (M/F), No. (\%) } & $\begin{array}{l}1,558(56.2) / 1,213 \\
\quad(43.8)\end{array}$ \\
\hline \multicolumn{2}{|l|}{$\begin{array}{l}\text { Karnofsky mean (SD) } \\
\text { [range] }\end{array}$} & $60.3(19.6)[10-100]$ \\
\hline \multirow[t]{12}{*}{ Primary tumor, No. (\%) } & Lung & $756(27.3)$ \\
\hline & Gastrointestinal & $478(17.2)$ \\
\hline & Breast & $280(10.1)$ \\
\hline & Pancreas & $271(9.8)$ \\
\hline & Urological & $175(6.3)$ \\
\hline & Prostate & $138(5.0)$ \\
\hline & Head-neck & $141(5.1)$ \\
\hline & Gynecologic & $126(4.5)$ \\
\hline & Liver & $94(3.4)$ \\
\hline & Hematological & $62(2.2)$ \\
\hline & Sarcoma & $46(1.7)$ \\
\hline & Others & 308 (11.1) \\
\hline \multirow[t]{2}{*}{ Disease, No. $(\%)$} & Loco-regional & $499(18.0)$ \\
\hline & Metastatic & $2,272(82.0)$ \\
\hline \multirow{2}{*}{$\begin{array}{l}\text { Anticancer treatment, } \\
\text { No. }(\%)\end{array}$} & Disease-oriented & $2,067(74.6)$ \\
\hline & Palliative Care & $585(21.1)$ \\
\hline \multirow[t]{5}{*}{ Place of visit, No. (\%) } & Outpatients & $761(27.5)$ \\
\hline & Day hospital & $297(10.7)$ \\
\hline & Home care & $502(18.1)$ \\
\hline & Hospice & $89(3.2)$ \\
\hline & Hospital inpatient & $1,122(40.5)$ \\
\hline \multirow[t]{4}{*}{ Setting, No. $(\%)$} & Palliative care & $623(22.5)$ \\
\hline & Oncology & $1,397(50.4)$ \\
\hline & Pain therapy & $738(26.6)$ \\
\hline & Radiotherapy & $13(0.5)$ \\
\hline $\begin{array}{l}\text { Mean background pain } \\
\text { intensity at } \mathrm{T} 0(\mathrm{SD})\end{array}$ & & $2.9(1.8)$ \\
\hline $\begin{array}{l}\text { Mean opioid doses } \\
\text { (SD), mg/d }\end{array}$ & & $81.3(95.7)$ \\
\hline
\end{tabular}

were expressed as mean $\pm \mathrm{SD}$, unless otherwise specified. Pearson's chi-square test and the Fisher exact test, as needed, were used for frequency analysis. To compare mean patient characteristic changes and their corresponding SDs, with $95 \%$ confidence intervals, the paired-samples Student $t$ test was used, with I type error set at $5 \%$. PGI was categorized into three classes: deterioration (PGI $\leq-1)$, no change $(\mathrm{PGI}=0)$, and improvement $(\mathrm{PGI} \geq 1)$. The level of pain intensity was categorized into three classes $($ mild $=1-3$, moderate $=$ $4-6$, severe $=7-10)$. Univariate analysis of variance (ANOVA) was performed to evaluate difference between patients' clinical characteristics, and post hoc analysis with the Bonferroni test was used to determine whether there were pairwise differences. Multivariate logistic regression analysis was performed on the significant variables using ANOVA to evaluate the correlation between patient characteristics (independent variables) and PGI groups (dependent variables). Pearson correlation analysis was conducted to assess the association between PPGR and patient clinical variables. Data were analyzed by IBM SPSS software, version 22 (IBM Corp., Armonk, NY, USA). All $P$ values were two-sided, and $P<0.05$ was considered statistically significant.

\section{Results}

From the original study, 689 patients with pain at T0 and complete assessment at T7 were analyzed. The characteristics of these patients are reported in Table 1. The mean age (SD) was 66.7 (11.8) years, 354 patients $(51.4 \%)$ were males, and 396 patients $(57.5 \%)$ had a Karnofsky level of $\leq 50$. The mean Karnofsky level (SD) was 53.7 (12.9). The mean MDAS value (SD) was 4.2 (3.4). One hundred sixty-four patients $(23.8 \%)$ had an MDAS in the range of 7-12 at T0. Twenty-six patients $(3.8 \%)$ were CAGE positive.

The mean pain intensity (SD) was $5.98(2.4)$ at T0 and 3.36 (2.2) at T7. The mean difference (SD) was $2.62(2.2)$ points, which was statistically significant $(P<0.0005)$. At T0, $44.3 \%$ of patients reported severe pain intensity $(\geq 7 /$ 10 ), whereas only $8.1 \%$ of patients reported severe intensity at T7 $(P<0.0001$, chi-square test $)$.

\section{PPIG}

The majority of patients $(87.5 \%)$ indicated a PPIG of $\leq 3$ as a target. The mean PPIGs at T0 and T7 (SD) were 1.33 (1.59), and $0.91(1.23)$, respectively $(\Delta-0.42[1.36])$. The difference was statistically significant $(<0.0005)$. Eightythree patients $(12 \%)$ had a PPIG of $\geq 5$. A higher PPIG $(>4)$ was significantly associated with a lower Karnofsky level (odds ratio $[\mathrm{OR}]=0.97,95 \%$ confidence interval $[\mathrm{CI}]=0.95-0.98, P=0.002)$ and higher pain intensity at T0 $(\mathrm{OR}=1.61,95 \% \mathrm{CI}=1.41-1.83, P<0.0005)$.

\section{PGI}

Five hundred thirty-four patients $(77.5 \%$ reported an improvement in PGI [at least bit better]) (Table 2). Patients perceived an MCID (a bit better) with a mean decrease in pain intensity of -2.09 . A better improvement corresponded to a mean change of -3.41 , whereas a much better improvement corresponded to a mean change of 4.59 points on the pain intensity scale. In 143 patients (20.7\%), no changes (no improvement, no deterioration) were recorded. In a low number of patients, pain intensity worsened. Patients perceived an MCID (a little worse) with a mean increase in pain intensity of 0.25 . They perceived a worse with a mean increase of 2.33 points.

In the univariate analysis, pain intensity at T0, MDAS, and PPIG at T0 were related to PGI, categorized into three classes (no change, improvement, deterioration) (Table 3). Pain intensity at T7 was inversely correlated with PGI (the lower the pain intensity, the higher the PGI). In the multiple logistic regression analysis, higher pain intensity at T0 and lower pain intensity at T7 were independently related to PGI (Table 4).

\section{PPGR}

At T7, 207 (30.0\%) patients achieved their target (PPIG). PPGR was correlated with PPIG both at T0 and T7 and 
Table 2. Minimal clinical difference according to Patient Global Impression after comprehensive pain management

\begin{tabular}{lrrrrrrrr}
\hline \multicolumn{1}{l}{} & \multicolumn{2}{l}{ PGI } & & & & & & \\
\cline { 2 - 8 } ESAS Change Score & & Much Better & Better & A Bit Better & The Same & A Little Worse & Worse & Much Worse \\
\hline Pain & No. & 147 & 162 & 225 & 143 & 8 & 3 & 1 \\
& Mean (SD) & $-4.59(2.01)$ & $-3.41(1.86)$ & $-2.09(1.33)$ & $-0.84(1.84)$ & $0.25(1.75)$ & $2.33(0.58)$ & 0.0 \\
\hline
\end{tabular}

ESAS $=$ Edmonton Symptom Assessment System; PGI = Patient's Global Impression.

Table 3. ANOVA analysis

\begin{tabular}{|c|c|c|c|c|c|}
\hline & & \multicolumn{3}{|l|}{ PGI } & \multirow[b]{2}{*}{$P$} \\
\hline & & No Change & Improvement & Deterioration & \\
\hline Variables & No. & 143 & 534 & 12 & \\
\hline Age, y & Mean (SD) & $67.3(12.1)$ & $66.5(11.9)$ & $68.2(7.4)$ & 0.693 \\
\hline Karnofsky & Mean (SD) & $55.6(12.9)$ & $53.2(12.9)$ & $54.2(13.1)$ & 0.137 \\
\hline Pain T0 & Mean $(\mathrm{SD})$ & $4.6(2.7)$ & $6.4(2.1)$ & $4.3(2.1)$ & $\begin{array}{l}<0.0005 \\
1 \text { vs } 0 \\
1 \text { vs } 2\end{array}$ \\
\hline Pain T7 & Mean $(\mathrm{SD})$ & $3.8(2.9)$ & $3.2(1.9)$ & $5.1(1.9)$ & $\begin{array}{l}0.001 \\
1 \text { vs } 0 \\
1 \text { vs } 2\end{array}$ \\
\hline MDAS & Mean $(\mathrm{SD})$ & $3.8(3.2)$ & $4.4(3.4)$ & $2.1(2.6)$ & $\begin{array}{l}0.007 \\
1 \text { vs } 2\end{array}$ \\
\hline Patient goal T0 & Mean (SD) & $0.7(1.2)$ & $1.5(1.7)$ & $0.8(1.1)$ & $\begin{array}{l}<0.0005 \\
1 \text { vs } 0\end{array}$ \\
\hline Patient goal T7 & Mean (SD) & $0.7(1.1)$ & $0.9(1.3)$ & $1.0(1.2)$ & 0.061 \\
\hline
\end{tabular}

Patient Global Impression was categorized into three classes: no change (PGI $=0$ ), improvement $(\mathrm{PGI} \geq 1)$, deterioration $(\mathrm{PGI} \leq 1)$.

ANOVA $=$ analysis of variance; MDAS = Memorial Delirium Assessment Scale; PGI = Patient Global Impression.

Table 4. Patient Global Impression

\begin{tabular}{llllc}
\hline PGI & & OR & $95 \%$ CI & $P$ \\
\hline No change & PAIN T0 & 2.177 & $1.310-3.619$ & 0.003 \\
& PAIN T7 & 0.437 & $0.270-0.706$ & 0.001 \\
& MDAS & 1.227 & $0.955-1.577$ & 0.109 \\
& Patient pain goal T0 & 0.925 & $0.536-1.595$ & 0.778 \\
Improvement & PAIN T0 & 4.736 & $2.800-8.011$ & $<0.0005$ \\
& PAIN T7 & 0.217 & $0.132-0.356$ & $<0.0005$ \\
& MDAS & 1.260 & $0.978-1.622$ & 0.074 \\
& Patient pain goal T0 & 1.259 & $0.728-2.178$ & 0.409 \\
\hline
\end{tabular}

Multiple logistic regression in reference to PGI category of deterioration.

$\mathrm{CI}=$ confidence interval; MDAS $=$ Memorial Delirium Assessment Scale; $\mathrm{OR}=$ odds ratio; $\mathrm{PGI}=$ Patient Global Impression.

was inversely correlated to pain intensity recorded at $\mathrm{T} 0$ and T7 and a lower Karnofsky level (Table 5).

Patients with higher pain intensity at T0 had a favorable PGI $(P<0.0001)$, even when the target, based on PPIG response, was not achieved. No significant differences among categories of pain intensity were found for PPGR $(P>0.05)$ (Table 6).

\section{Discussion}

This subanalysis of data gathered in an international multicenter study, which recruited a large number of patients, provided interesting information to help physicians in personalizing pain management and realizing how much patients would like to improve their pain and how effectively physicians can help them achieve their target. Pain intensity significantly decreased after comprehensive palliative care treatment.

\section{PPIG}

Most patients indicated a PPIG of $\leq 3$, confirming existing data from previous studies [16]. Although in these studies PPIG remained unchanged at undetermined follow-up visits $[9,16,17]$, in the present study, PPIG decreased after one week, as if patients wanted to raise their expectations once they had an improvement in pain intensity or after achieving their initial target. The long follow-up period of these studies and the short and acute period of the present study could explain the differences.

\section{PGI}

In most patients PGI for pain was positive, given that 534 patients reported an improvement in pain intensity after one week of treatment. One week is considered to be a meaningful time frame to stabilize patients admitted to a place like a palliative care unit, where efforts to manage symptoms are intensive and effective [18]. Patients perceived an MCID with a decrease in pain intensity of about 2 points. For perceiving a better improvement, patients required a decrease in pain intensity of about 3.5. A much better improvement was perceived with a mean decrease of 4.5 points. In previous studies, a lower 
Table 5. Factors correlated with Patient Pain Goal Response

\begin{tabular}{|c|c|c|}
\hline & & Patient Pain Goal Respons \\
\hline \multirow[t]{3}{*}{ Age } & Pearson correlation & 0.028 \\
\hline & $P$ (2-tailed) & 0.460 \\
\hline & No. & 686 \\
\hline \multirow[t]{3}{*}{ Gender } & Pearson correlation & -0.024 \\
\hline & $P(2$-tailed $)$ & 0.527 \\
\hline & No. & 686 \\
\hline \multirow[t]{3}{*}{ Karnofsky } & Pearson correlation & $-0.151 * *$ \\
\hline & $P($ (2-tailed) & $<0.0005$ \\
\hline & No. & 683 \\
\hline \multirow[t]{3}{*}{ Pain T0 } & Pearson correlation & $-0.272 * *$ \\
\hline & $P(2$-tailed $)$ & $<0.0005$ \\
\hline & No. & 686 \\
\hline \multirow[t]{3}{*}{ Patient pain goal T0 } & Pearson correlation & $0.508 * *$ \\
\hline & $P(2$-tailed $)$ & $<0.0005$ \\
\hline & No. & 686 \\
\hline \multirow[t]{3}{*}{ Pain T7 } & Pearson correlation & $-0.778 * *$ \\
\hline & $P(2$-tailed $)$ & $<0.0005$ \\
\hline & No. & 686 \\
\hline \multirow[t]{3}{*}{ Patient pain goal T7 } & Pearson correlation & $0.115^{* *}$ \\
\hline & $P(2$-tailed) & 0.003 \\
\hline & No. & 686 \\
\hline
\end{tabular}

Table 6. Patient Pain Goal Response and Patient Global Impression, according to the categories of pain intensity measured at T0

\begin{tabular}{llclc}
\hline & Mild, & Moderate, & Severe, & \\
& No. $(\%)$ & No. $(\%)$ & No. $(\%)$ & Total \\
\hline Pain T0 & 127 & 257 & 305 & 689 \\
PPGR $(\geq 1)$ & $42(33.1)$ & $77(30.0)$ & $85(27.9)$ & 204 \\
PGI $(\geq 1)$ & $53(41.7)$ & $215(83.6) *$ & $266(87.2)^{*}$ & 534 \\
\hline
\end{tabular}

PGI $=$ Patient Global Impression; PPGR $=$ Patient Pain Goal Response.

* $P<0.0001$ in respect to mild pain intensity.

MCID was found $[7,12]$. The retrospective nature of design, the longer and not constant intervals among observations, the use of different categories anchored to the well-being scale [11], or the setting (radiotherapy, outpatients), may explain the differences. Some studies have reported more relevant changes of pain intensity to perceive a meaningful clinical benefit, including a 33\% decrease or a reduction of two or more points [19,20], confirming data found in this study.

The factors principally related to improvement in PGI have never been assessed. In this study, the higher the pain intensity, the better the PGI, although not all patients were able to achieve their target. It is likely that a more evident feeling of improvement perceived by patients when they perceive a net decrease in pain intensity (about halving the pain intensity with about 2.5 points of difference) explains this finding. Similarly, higher PPIG was also independently associated with a positive PGI, possibly because the level expectation is easier to be reached with just a little change in pain intensity. Thus, patients who accept a higher level of pain intensity are more likely to achieve better satisfaction.
Eighty-three patients $(12 \%)$ had higher values of PPIG at admission. These patients are likely to require only minimal changes in pain intensity for a positive PGI. On the other hand, a low level of pain intensity at T7 was associated with a better PGI, as a consequence of adequate pain management.

In some studies, initial pain intensity has been found to be a negative factor for pain prognosis [16,21-23]. These studies, however, clearly showed that clinical undertreatment was responsible for the outcome. In fact, patients were stabilized for a very long time (one to three weeks), suggesting a nonoptimized method for opioid dose titration. In other circumstances, a retrospective long follow-up in outpatients (three weeks), based on only one therapeutic intervention, would have biased the outcome. Indeed, pain intensity is a dynamic concept, depending on the moment in which the patient is intercepted [18]. Several surveys and also daily practice suggest that pain control is commonly achieved in a few days in the majority of patients by using personalized opioid dose titration [24-30]. Data from this study confirm that the higher the level of initial pain intensity, the better the patient satisfaction score after proper pain management.

\section{PPGR}

Thirty percent of patients achieved their target (PPIG) after proper pain management. This percentage was lower than that of patients with a positive PGI. In patients with higher pain intensity or a lower Karnofsky level, PPGR was more likely to be achieved, allowing the patient to reach the level of PPIG expected. Thus, a higher PPIG allowed the achievement of a better PPGR, as small improvements were sufficient to obtain the target level desired by patients. This finding reflects the features of PGI. Patients with higher pain intensity may have lower expectations when rating their PPGI scores as high, which means that active pain management may have a greater opportunity to achieve PPGR. Patients with a lower Karnofsky status may have a more positive impression after palliative care treatment or may merely have lower expectations. This observation deserves further study.

This study has some limitations. In comparison with previous trials examining issues regarding clinical changes as perceived by patients and PPIG, data were obtained from patients recruited in palliative care units, where it is likely that symptom assessment and treatments are more intensive. Thus, these data are not generalizable to outpatients or home care settings. A PPIG scale was used to test MCID in this study. That is the way patients may individually perceive clinical change. This tool proved to be repeatable and easy to understand, even though it lacks other external criteria. 


\section{Conclusions}

The PPIG allows clinicians to individualize patient care and ensures intrapatient determination of a practical and meaningful pain response. The PPIG, PGI, and PPGR are measurements that are relevant to the assessment and decision-making process, according to patients' expectations. Some factors, such as pain intensity, PPIG, and Karnofsky, may influence clinical response, assessed by PGI and PPGR. Further studies should investigate these aspects in other palliative care settings.

\section{References}

1. Portenoy RK, Ahmed E. Cancer pain syndromes. Hematol Oncol Clin North Am 2018;32(3):371-86.

2. Raj SX, Thronaes M, Brunelli C, et al. A crosssectional study on prevalence of pain and breakthrough pain among an unselected group of outpatients in a tertiary cancer clinic. Support Care Cancer 2014;22(7):1965-71.

3. Hui D, Bruera E. The Edmonton Symptom Assessment System 25 years later: Past, present, and future developments. J Pain Symptom Manage 2017; 53(3):630-43.

4. Lydick E, Epstein R. Interpretation of quality of life changes. Qual Life Res 1993;2(3):221-6.

5. Maringwa J, Quinten C, King M, et al. Minimal clinically meaningful differences for the EORTC QLQ-30 and EORTC QLQ-BN20 scales in brain cancer patients. Ann Oncol 2011;22(9):2107-12.

6. Norman GR, Sloan JA, Wyrwich KW. Interpretation of changes in health-related quality of life: The remarkable universality of half a standard deviation. Med Care 2003;41(5):582-92.

7. Bedart G, Zeng L, Zhang L, et al. Minimal clinically important differences in the Edmonton Symptom Assessment System in patients with advanced cancer. J Pain Symptom Manage 2013;46:192-200.

8. Lauridsen HH, Hartvigsen J, Manniche C, et al. Responsiveness and minimal clinically important difference for pain and disability instruments in low back pain patients. BMC Musculoskelet Disord 1006;7:82.

9. Dalal S, Hui D, Nguyen L, et al. Achievement of personalized pain goal in cancer patients referred to a supportive care clinic at a comprehensive cancer center. Cancer 2012;118(15):3869-77.

10. Hui D, Park M, Shamieh O, et al. Personalized symptom goals and response in patients with advanced cancer. Cancer 2016;122(11):1774-81.

11. Hui D, Bruera E. Minimal clinically important differences in the Edmonton Symptom Assessment System: The anchor is key. J Pain Symptom Manage 2013;45 :e4-5.

12. Hui D, Shamieh O, Paiva CE, et al. Minimal clinically important differences in the Edmonton Symptom
Assessment Scale in cancer patients: A prospective, multicenter study. Cancer 2015;121(17):3027-35.

13. Mercadante S, Adile C, Lanzetta G, et al. Personalized symptom goals and patient global impression on clinical changes in advanced cancer patients. Oncologist 2019;24(2):239-46.

14. Breitbart W, Rosenfeld B, Roth A, et al. The Memorial Delirium Assessment Scale. J Pain Symptom Manage 1997;13(3):128-37.

15. Parsons HA, Delgado-Guay MO, El Osta B, et al. Alcoholism screening in patients with advanced cancer: Impact on symptom burden and opioid use. J Palliat Med 2008;11(7):964-8.

16. Arthur J, Tanco K, Park M, et al. Personalized pain goal as an outcome measure in routine cancer pain assessment. J Pain Symptom Manage 2018;56(1):80-7.

17. Yennurajalingam S, Kang JH, Hui D, et al. Clinical response to an outpatient palliative care consultation in patients with advanced cancer and cancer pain. J Pain Symptom Manage 2012;44(3):340-50.

18. Mercadante S, Gebbia V, David F, et al. Does pain intensity predict a poor opioid response in cancer patients? Eur J Cancer 2011;47(5):713-7.

19. Farrar JT, Portenoy RK, Berlin JA, et al. Defining the clinically important difference in pain outcome measures. Pain 2000;88(3):287-94.

20. Farrar JT, Pritchett YL, Robinson M, et al. The clinical importance of changes in the 0 to 10 numerical rating scale for worst, least, and average pain intensity: Analyses of data from clinical trials of duloxetine in pain disorders. J Pain 2010;11(2):109-18.

21. Apolone G, Corli O, Caraceni A, et al. Pattern and quality of care of cancer pain management. Results from the Cancer Pain Outcome Research Study Group. Br J Cancer 2009;100(10):1566-7.

22. Fainsinger R, Fairchild A, Nekolaichuk C, Lawlor P, Lowe $\mathrm{S}$, Hanson J. Is pain intensity a predictor of the complexity of cancer pain management? J Clin Oncol 2009; 7:585-90.

23. Knudsen AK, Brunelli C, Klepstad P, et al. Which domains should be included in a cancer pain classification system? Pain 2012;153(3):696-703.

24. Hwang S, Chang V, Fairclough D, Kasimis B. Development of a cancer pain prognostic scale. J Pain Symptom Manage 2002;24(4):366-78.

25. Kang JH, Kwon JH, Hui D, Yennurajalingam S, Bruera E. Changes in symptom intensity among cancer patients receiving outpatient palliative care. J Pain Symptom Manage 2013;46(5):652-60.

26. Mercadante S, Porzio G, Adile C, et al. Pain intensity as prognostic factor in cancer pain management. Pain Pract 2015;15(1):E1-8.

27. Mercadante S, Gebbia V, David F, et al. Tools for identifying cancer pain of predominantly neuropathic origin and opioid responsiveness in cancer patients. J Pain 2009;10(6):594-600. 
28. Mercadante S, Adile C, Caruselli A, et al. The palliative-supportive care unit in a comprehensive cancer center as crossroad for patients' oncological pathway. PLoS One 2016;11(6):e0157300.

29. Chang V, Hwang SS, Kasimis B. Longitudinal documentation of cancer pain management outcomes: A pilot study at a VA medical center. J Pain Symptom Manage 2002;24(5):494-505.

30. Stromgren A, Groenvold M, Petersen M, et al. Pain characteristics and treatment outcome for advanced cancer patients during the first week of specialized palliative care. J Pain Symptom Manage 2004;27:104-13. 